\title{
RELACIÓN ENTRE LOS GASTOS E INGRESOS DEL GOBIERNO EN ESPAÑA, 1958-2014*
}

\author{
Recibido: 25 de mayo de 2017 • Aprobado: 03 de septiembre de 2017 \\ DOI: $10.22395 /$ seec.v20n45a1
}

\author{
Manuel Jaén-García**
}

\section{RESUMEN}

En este artículo se utiliza metodología de cointegración y causalidad en series temporales para analizar la relación ingreso-gasto, gasto-ingreso en la economía española durante el período 1958-2014. Se ha tenido en cuenta el posible cambio estructural en ambas series durante el período objeto de estudio, así como la posible existencia de causalidad tanto a corto como a largo plazo. Los resultados muestran causalidad en la dirección ingreso a gasto, pero no al contrario. Dada la importancia en España de la reducción del déficit público se infiere que es necesario ajustar a la baja el gasto público con el consiguiente perjuicio que se puede producir en el crecimiento económico español.

\section{PALABRAS CLAVE}

Gasto público; ingreso público; política fiscal; cambio estructural; cointegración; causalidad; España.

\section{CLASIFICACIÓN JEL}

H00, E21, E62

\section{CONTENIDO}

Introducción. 2. Hipótesis del debate impuesto-gasto. 3. Revisión de la literatura empírica. 4. Evolución reciente de las finanzas públicas españolas. 5. Contrastación empírica del modelo. 6. Conclusiones. Bibliografía

\footnotetext{
Este trabajo es producto del proyecto de investigación "La senda del gasto público en España. Un nuevo enfoque" desarrollado durante el período 2014-2016. Financiado por el Instituto de Estudios Fiscales (Ministerio de Hacienda y Administración Pública de España), Grupo de Investigación Avances en Economía Aplicada de la Universidad de Almería, España.

** Licenciado en Matemáticas, Universidad de Granada, Granada, España. Licenciado en Ciencias Económicas y Empresariales Universidad de Málaga, Málaga, España. Doctor en Ciencias Económicas y Empresariales, Universidad de Granada, Granada, España. Catedrático de Economía Aplicada, Universidad de Almería, Almería, España. Director del Grupo de Investigación Avances en Economía Aplicada, Departamento de Economía y Empresa, Universidad de Almería, Almería, España, Teléfono +34 950 015197, Fax +34 950 015172, Dirección: Cañada de S/Urbano 04120 Almería, España. Correo electrónico:mjaen@ual.es.
} 


\section{RELATIONSHIP BETWEEN GOVERNMENT EXPENSES AND REVENUES IN SPAIN, 1958-2014}

ABSTRACT

This article uses co-integration and causality methodology in time series to analyze the relationship between income and expenditure, expenditure and income in the Spanish economy during the period 1958-2014. The possible structural change in both series during the period under study has been taken into account, as well as the possible existence of both short-term and long-term causality. The results show causality in the direction of revenue to expenditure, but not the other way around. Given the importance of reducing the public deficit in Spain, it states that it is necessary to adjust public expenditure downwards with the consequent damage that may occur in Spain's economic growth.

\section{KEYWORDS}

Public expenditure; public revenue; fiscal policy; structural change; co-integration; causality; Spain.

\section{JEL CLASSIFICATION}

H00, E21, E62

\section{CONTENT}

Introduction. 2. Hypothesis of the tax-expenditure debate. 3. Review of empirical literature. 4. Recent developments in Spanish public finances. 5. Empirical contrast of the model. 6. Conclusions. Bibliography.

\section{RELAÇÃO ENTRE OS GASTOS E INGRESSOS DO GOVERNO NA ESPANHA, 1958-2014}

\section{RESUMO}

Neste artigo, utiliza-se metodologia de cointegração e causalidade em séries temporais para analisar a relação ingresso-gasto, gasto-ingresso na economia espanhola durante o período 1958-2014. Consideraram-se a possível mudança estrutural em ambas as séries durante o período em estudo bem como a possível existência de causalidade tanto em curto quanto em longo prazo. Os resultados mostram causalidade na direção ingresso a gasto, mas não ao contrário. Tendo em vista a importância, na Espanha, da redução do déficit público, infere-se que seja necessário ajustar à baixa o gasto público com o consequente prejuízo que pode ser produzido no crescimento econômico espanhol.

\section{PALAVRAS-CHAVE}

Gasto público; ingresso público; política fiscal; mudança estrutural; cointegração; causalidade; Espanha.

\section{CLASSIFICAÇÃO JEL} H00, E21, E62

\section{CONTEÚDO}

Introdução. 2. Hipótese do debate imposto-gasto. 3. Revisão da literatura empírica. 4. Evolução recente das finanças públicas espanholas. 5. Contraste empírico do modelo. 6. Conclusões. Bibliografia. 


\section{INTRODUCCIÓN}

La relación ingresos-gastos ${ }^{1}$ ha sido objeto de amplia investigación empírica desde 1995. El análisis se ha realizado tanto a escala nacional como estatal o municipal. Se han usado diferentes técnicas de raíces unitarias, cointegración y causalidad tanto con datos de series temporales como con datos de panel.

Determinar cuál de las hipótesis caracteriza de forma más adecuada una economía es más que un ejercicio intelectual por cuanto puede contribuir al descubrimiento de una solución al problema de los crecientes déficits presupuestarios.

Esta es una cuestión del mayor interés en la Unión Económica y Monetaria Europea (UEM), pues tanto el tratado que la constituye como el Pacto de Estabilidad y Crecimiento (PEC) tienen como puntos centrales la tendencia al equilibrio presupuestario y el mantenimiento de la deuda pública, en relación con el Producto Interno Bruto (PIB), por debajo de unos límites preestablecidos.

Se analizan y contrastan las cuatro hipótesis. Se usan datos de ingreso, gasto público y PIB para España en el período 1958-2014. A diferencia de trabajos anteriores, por ejemplo Castro (2002), se ha tenido en cuenta la posible presencia de cambios estructurales en los datos, lo que nos lleva a utilizar contrastes de raíces unitarias, cointegración y causalidad Granger que consideren esa circunstancia.

Los resultados nos indican que existe causalidad en la dirección ingresos a gastos. Este resultado tiene importantes implicaciones en cuanto a la política fiscal a llevar a cabo por el Gobierno, pues un aumento (disminución) del ingreso puede llevar a un aumento (disminución) del gasto.

En el primer apartado se discuten las cuatro hipótesis, y se presentan algunos contrastes empíricos en el segundo; en el tercero se analiza la evolución reciente de las finanzas públicas en España; en el cuarto se realiza la contrastación empírica para el caso español y en el quinto se presentan las conclusiones.

\section{HIPÓTESIS DEL DEBATE IMPUESTO-GASTO}

De acuerdo con Payne (2003), la literatura gasto-impuesto ha considerado cuatro hipótesis en cuanto a la relación intertemporal entre gastos e ingresos públicos.

Primero, la hipótesis impuesto-gasto tal como fue formulada por Friedman (1978), argumenta que aumentar los impuestos, con el propósito de incrementar los recur-

\footnotetext{
1 La literatura utiliza indistintamente los términos ingreso-gasto (revenue-expenditure o revenue-spend), impuesto-gasto (tax-expenditure) e, incluso, ingreso impositivo-gasto (tax revenue-expenditure).
} 
sos disponibles por el sector público y reducir el déficit presupuestario da como resultado una subida del gasto público. En efecto, si los ingresos tienen un efecto positivo en los gastos, entonces las reducciones en los ingresos repercutirán en reducciones en los gastos y viceversa, de forma que no se producirá una reducción en el déficit presupuestario.

Un punto de vista alternativo de esta hipótesis es el de Wagner (1976), así como Buchanan-Wagner (BW, 1977) que sugieren que los aumentos de los ingresos darán lugar a una bajada en los gastos vía ilusión fiscal. Dentro de este esquema, los gastos públicos financiados por medios diferentes de la imposición directa tienen como resultado que la percepción del contribuyente sobre el precio de dichos gastos sea menor que la que sería bajo dicha imposición directa. Aunque el contribuyente puede pagar menos en términos de imposición directa, la ilusión fiscal surge en que el contribuyente paga a través de imposición indirecta, vía elevados tipos de interés de la deuda, con lo que para la economía en su conjunto se genera un efecto sustitución, así como inflación que lleva a deslizamientos en el tramo impositivo.

El punto de vista de BW se centra en que el gasto público es financiado a partir de tres fuentes: imposición directa, emisión de deuda e imposición indirecta a través de la inflación. Desde una perspectiva política, el nivel óptimo de fuentes de ingresos para financiar un gasto dado depende de sus costos relativos. Así, en el contexto del esquema BW, un aumento de los impuestos (imposición directa) puede llevar a una reducción en el gasto público si hay una reducción en las formas de imposición indirecta, es decir, una reducción en la emisión de deuda, así como en los deslizamientos en el tramo impositivo. De forma alternativa, una reducción en los ingresos puede llevar al crecimiento del gasto cuando los consumidores demandan más programas de gasto.

Aunque la hipótesis señala causalidad unidireccional de los ingresos públicos a los gastos públicos, el signo del coeficiente de la ecuación que relaciona ingresos con gastos será positivo en el primer caso, y negativo, en el segundo.

Segundo, la hipótesis gasto-impuesto sugiere que las decisiones de gasto son, en principio, determinadas con ajustes en la política impositiva y en los ingresos para hacer frente a la demanda de gasto. Dada la proposición de equivalencia ricardiana de que lo que los gobiernos piden prestado hoy tiene como resultado una subida en las obligaciones impositivas futuras que se capitaliza por los contribuyentes, Barro (1979) cuestiona la hipótesis de ilusión fiscal considerada en BW. En el contexto del esquema de equivalencia ricardiana, los aumentos en el gasto público llevan a incrementos en los impuestos. Esa subida impositiva puede ser contemporánea 
al aumento del gasto o bien puede existir un desfase entre ambos. Lo importante es que si se admite una cierta endogeneidad en el comportamiento del Gobierno, el cumplimiento de la restricción presupuestaria intertemporal del Gobierno (RPIG) da soporte a la hipótesis de que los gastos determinan los ingresos. Entre otros Roberts (1978), así como Peacock y Wiseman (1979) proponen que las subidas temporales en el gasto público debidas a situaciones de crisis se trasladan a mayores impuestos de forma permanente (es la hipótesis del efecto desplazamiento). Así, bajo la hipótesis gasto-impuesto, reducciones en los gastos públicos llevarán a reducciones en los déficits, de forma que el control del déficit puede lograrse a través de aumentos imprevistos en la presión fiscal o de límites (legales) estrictos en el nivel del gasto público.

Tercero, la hipótesis de sincronización fiscal argumenta que las decisiones sobre los ingresos y los gastos se adoptan de forma simultánea. Musgrave (1966) y Meltzer y Richard (1981) sugieren que los votantes comparan los beneficios y costos marginales de los programas de Gobierno cuando deciden el nivel apropiado de gastos e ingresos públicos. Este punto de vista es apoyado por el modelo de suavización impositiva de Barro (1979) que está basado en el argumento de la equivalencia ricardiana. Los superávits o déficits fiscales pueden producirse bajo esta circunstancia porque los gobiernos tienen diferentes objetivos de política económica, incluso, aunque las decisiones de ingresos y gastos se muevan juntas.

Cuarto, la hipótesis de separación institucional o neutralidad fiscal sostiene que las decisiones en imposición son independientes de la asignación de los gastos públicos. Según Wildavsky (1988) y Baghestani y McNown (1994) los gastos son determinados por los requerimientos de los ciudadanos, y los ingresos dependerán de la cantidad máxima de impuestos que los ciudadanos puedan soportar. En consecuencia, es solo cuestión de coincidencia que se pueda lograr el equilibrio fiscal. Esto es posible, de acuerdo con Obeng (2015), porque el crecimiento económico asegura que, en el largo plazo, las instituciones se separan y se independizan cada vez más por lo que es posible que las decisiones de ingresos y gastos se tomen de forma separada.

\section{REVISIÓN DE LA LITERATURA EMPÍRICA}

La literatura sobre este tema ha producido una amplia variedad de resultados debido, en parte, a las diferentes aproximaciones metodológicas, al grado de agregación temporal, así como a los períodos examinados. Aunque la metodología específica varía en los diferentes estudios, la literatura empírica se centra en el concepto de causalidad en el espíritu de Granger. En este contexto, se apoya la versión de Friedman de la hipótesis impuesto-gasto, si la causalidad unidireccional es evidente de 
ingresos a gastos y los ingresos tienen un impacto positivo en los gastos. Se cumple la versión BW si existe causalidad unidireccional de ingresos a gastos y los ingresos tienen un impacto negativo en los gastos. Se apoya la versión gasto-impuesto, si la causalidad va de gastos a ingresos. Mientras que se apoya la sincronización fiscal si existe causalidad bidireccional entre ingresos y gastos. Por último, la ausencia de causalidad apoya la hipótesis de separación institucional.

Los trabajos realizados se pueden dividir grosso modo entre trabajos que realizan el análisis a escala nacional y aquellos que consideran la escala estatal o local. Entre los primeros la mayoría de los trabajos se han realizado para Estados Unidos de América (EUA) y los principales países europeos, y un número menor para los países industrializados del área del Pacífico y los países menos desarrollados. Se pueden citar Darrat (2002) para Líbano y Túnez; Chang, Liu y Caudill, (2002) para diez países entre ellos Japón, Reino Unido (UK) y EUA; Reddick y Hassan (2003) para Canadá, UK y EUA; Kollias y Paleologou (2006) para los 15 primeros países miembros de la Unión Europea(UE); Chang y Chiang (2009) para un panel de 15 miembros de la Organización de Cooperación y desarrollo económico (OCDE); Saysombath y Kyophilavong (2013) para Laos. Entre los segundos (análisis a escala estatal o local) la gran mayoría utiliza datos de panel. Es el caso de Dahlberg y Johansson (1998) para los municipios suecos; Moisio (2001) para los municipios finlandeses; Reddick (2002) para las provincias canadienses; Hou y Huand (2009) para las provincias chinas; Jaén (2011) para las comunidades autónomas españolas; Saunoris (2015) para un panel de 48 estados de EUA.

Hay varias diferencias clave entre el análisis a escala subnacional y a escala nacional. Primero, los gobiernos estatales y locales no tienen la posibilidad de llevar a cabo políticas inflacionarias como un medio de aumentar los impuestos como es el caso de los gobiernos nacionales. Segundo, muchos gobiernos estatales y locales gobiernan bajo requerimientos legislativos y constitucionales que intentan restringir los déficits presupuestarios. Tercero, los presupuestos de los gobiernos estatales y locales están influidos por elevados niveles de subvenciones gubernamentales. La presencia de subvenciones como una fuente de financiación puede afectar los gastos (efecto flypaper) así como los ingresos (efecto sustitución). Por ejemplo, los cambios en los gastos pueden ser atribuidos a cambios en los ingresos cuando, de hecho, deben atribuirse a cambios en las subvenciones.

\section{EVOLUCIÓN RECIENTE DE LAS FINANZAS PÚBLICAS ESPAÑOLAS}

Desde 1976, el gasto público en España ha experimentado un fuerte crecimiento, tanto a precios corrientes como a precios constantes o como porcentajes del PIB. 
Durante el período 1958-2014, se pueden distinguir, según Tamames (2005), González (2013) y García (2015), tres fases distintas de acuerdo con su naturaleza con respecto al gasto público. La primera fase dura desde 1958 hasta 1975; en ella, el gasto público en términos reales era menor al 25\% del PIB. La intervención del Estado en la economía se llevaba a cabo a través de regulación en la forma de leyes y estatutos. En la década de 1970, la industrialización ayudó al crecimiento del gasto público. En la línea de la tradición wagneriana, el desarrollo económico fue de la mano de un aumento en la población, la educación, la urbanización, la salud y la redistribución del ingreso.

En el segundo período, entre 1976 y 1985, se produjo una rápida expansión del gasto público en España. Dicho gasto aumentó del 23,19\% del PIB al 42,5\%, en línea con los países de la OCDE, cuyo promedio de gasto representaba el $47 \%$ del PIB. Los investigadores están de acuerdo en que las fuerzas fundamentales que impulsaron el crecimiento del gasto en este período fueron: 1) la transición a la democracia que produjo un boom en las demandas de la sociedad que habían sido contenidas por el régimen franquista; 2) la crisis económica que forzó al Estado a conceder subvenciones a las empresas en forma de transferencias de capital; 3) la persistencia de los déficits presupuestarios y su nuevo modelo de financiación en el que se sustituye el recurso del señoreaje, a través del Banco de España, por la emisión de deuda pública y su negociación en los mercados, y 4) la descentralización de algunos gastos a través del nuevo modelo autonómico sin la contrapartida de corresponsabilidad fiscal de las comunidades autónomas.

Al mismo tiempo, la crisis económica provocó un aumento en el desempleo que se palió mediante el cobro de seguro de desempleo por parte de los trabajadores y, en menor medida, la jubilación anticipada con cargo a los fondos de la Seguridad Social de trabajadores de industrias en declive tal como la industria pesada de altos hornos, acerías y astilleros.

En el tercer período 1986-1993, el crecimiento del gasto fue determinado por la política del nuevo gobierno del Partido Socialista Obrero Español (PSOE) con la puesta en marcha de un Estado de bienestar en España. La universalización de la sanidad pública y el aumento del número de pensionistas a través de las pensiones no contributivas, así como el incremento de la cuantía de las pensiones contribuyeron a un mayor gasto público. De la misma forma, la educación se hizo obligatoria hasta los 16 años y se realizaron acuerdos con el sistema privado de educación que recibió subvenciones del Estado para garantizar la gratuidad de la educación en el tramo de edad entre los 6 y los 16 años. Por último, se produjo la consolidación de las comunidades autónomas que recibían cada vez un mayor número de transferencias del Estado central con las consiguientes necesidades de gasto. Todo ello 
provocó un fuerte aumento en el gasto con deterioro del equilibrio presupuestario. En la última fase de este período, la absoluta necesidad de reunir los requerimientos fijados por el tratado de Maastrich obligó a una reducción del déficit después de haber alcanzado en 1993 su valor más elevado en relación con el PIB (5,1\%).

Entre 1994 y 1998 se produjo un drástico cambio en el sector público español como una respuesta al cambio de paradigma económico desde el modelo social-demócrata poskeynesiano al sistema liberal-neoclásico. Las razones básicas para este cambio se encuentran en la necesidad de cumplir en su totalidad las condiciones del tratado de Maastrich (TM), así como el Pacto de Estabilidad y Crecimiento (PEC); en conjunción con que este período coincide con el principio de la fase ascendente del ciclo económico en 1996. Los elevados déficits presupuestarios (7,3\% del PIB en 1994) caen al $-2,9 \%$ en 1998 con lo que se cumplen las condiciones del PEC (-3\%).

Entre 1999 y 2007 se produce la consolidación de las finanzas públicas en España empujadas por el PEC, necesario para propiciar la entrada de España en la Unión Económica y Monetaria (2002), y la burbuja inmobiliaria que hizo posible un crecimiento sin precedentes. De forma que en 2001 se logra un presupuesto equilibrado, aunque el gasto público supone el $40 \%$ del PIB con una subida del $4 \%$ con respecto al promedio del período 1997-2001.

El período de la burbuja posibilitó el crecimiento del empleo unido a un aumento de la venta de viviendas lo que dio lugar a un incremento de la recaudación fiscal tanto directa, vía Impuesto de la Renta de las Personas Físicas (IRPF), por el incremento del empleo tanto en el sector de la construcción como en otros sectores, e indirecta debido a la recaudación por Impuesto del Valor Agregado (IVA) de la venta de vivienda nueva y el impuesto de transmisiones patrimoniales en la venta de vivienda usada. Por otra parte, aumentó la recaudación en impuestos locales derivados de la construcción de viviendas.

De 2007 a 2014 se produce una fuerte caída en la economía española debido al pinchazo de la burbuja inmobiliaria. Hasta 2008, la expansión continuó y el PIB español alcanzó el promedio de la UE 27. En ese momento, la economía española, arrastrada por la caída en las economías estadounidenses y europeas y sus propios problemas internos, entró en una profunda recesión de la que empezó a emerger en 2014 con crecimiento positivo del PIB, aunque modesto $(1,2 \%$ y con déficits presupuestarios del 7\% en 2013 y 6\% en 2014.

La evolución de los ingresos públicos es paralela a la de los gastos. Hasta la reforma iniciada en 1977, el sistema tributario español responde a lo que se denomina un sistema latino basado en el gravamen del producto de determinados objetos, tales como los bienes rústicos o urbanos, propiedad mobiliaria o actividades 
empresariales donde se combinan trabajo, capital y prestación de servicios. Esta imposición se complementa con impuestos sobre la renta. En 1986 con la entrada de España en la Unión Europea se pone en vigor el impuesto sobre el valor agregado (IVA) que sustituye al antiguo impuesto en cascada que gravaba en su totalidad cada una de las transacciones.

Hasta la mencionada reforma de 1977 existía un fuerte predominio de la imposición indirecta sobre la directa. Así, en 1958 la imposición directa suponía un 71,7\% de la indirecta, aunque la tendencia era descendente de forma que en 1963 el porcentaje era el 55\%. A partir de la reforma de 1964 se acentúa el predominio de los impuestos indirectos con un valor casi constante del $47,5 \%$. Sin embargo, la reforma de 1978 cambia los términos. En 1979 el porcentaje era del 92,5\% con un fuerte aumento de la recaudación por imposición directa. Esta tendencia se mantiene de manera que, en 1993, el porcentaje era del 124,2\%. En la actualidad la imposición indirecta supone el 27,6\% del total.

De acuerdo con Serrano y Bandrés (2015), hasta estas reformas la fiscalidad española no respondía a patrones europeos: la presión fiscal era muy inferior y su estructura distinta. Con el cambio ganó en equidad, flexibilidad, neutralidad y transparencia, y se asimiló a la vigente en los países comunitarios.

En la actualidad, los ingresos de las administraciones públicas españolas se nutren sobre todo de tres partidas: las cotizaciones sociales que suponen el 37\% del total de ingresos públicos; los impuestos directos que suponen el $28 \%$, y los indirectos que suponen el 28\%.

En conjunto, la presión fiscal en la economía española alcanzó un valor del 40,9\% del PIB en 2007, similar al promedio de la OCDE y tres puntos por debajo de la media europea. En 2011, la situación de crisis de la economía española, en el marco de la crisis europea, supuso una disminución de los ingresos, situándose estos en el 36,1\% del PIB. En 2014 se ha producido un ligero repunte los ingresos públicos (38,9\% del PIB).

Esta disminución impositiva, junto con el aumento del gasto público, supuso un aumento del déficit público que pasó de 1,9\% del PIB de superávit en 2007 al 9,6\% de déficit en 2011, con un máximo en 2009 del 11,0\% del PIB. La austeridad del último Gobierno situó el déficit, en 2014, en el 6\% del PIB.

\section{CONTRASTACIÓN EMPÍRICA DEL MODELO}

La contrastación empírica para el nexo ingresos-gastos para España se realiza con la metodología de cointegración y causalidad en series temporales. 
Las series utilizados son gasto público (GP), ingreso público (IP) y Producto Interno Bruto (PIB) todos en euros constantes del año 1995. Los datos han sido tomados de las bases de datos del Instituto Nacional de Estadística (INE), para el PIB, y de la Intervención General de la Administración del Estado (IGAE), para Gasto, Ingreso público y Déficit. Para unir las distintas series se ha utilizado el link legal del INE. Se han utilizado los deflactores correspondientes a cada serie y tomado los datos para el período 1958-2014².

El siguiente gráfico muestra la evolución del ingreso y el gasto públicos para el período considerado. Aunque ambas variables muestran trayectorias ascendentes bastante parecidas, y se solapan en ocasiones, se observan ciertos desfases entre ambas a partir de la existencia de un régimen democrático en España. Las fases de fuerte crecimiento de la economía, finales de los años noventa y los del boom en el sector de la construcción, traen consigo cortos superávits presupuestarios, aunque la tónica general es de continuados déficits en el período democrático.

\section{Gráfico 1. Gastos e ingresos públicos en euros ctes de 1995 y \% del PIB}

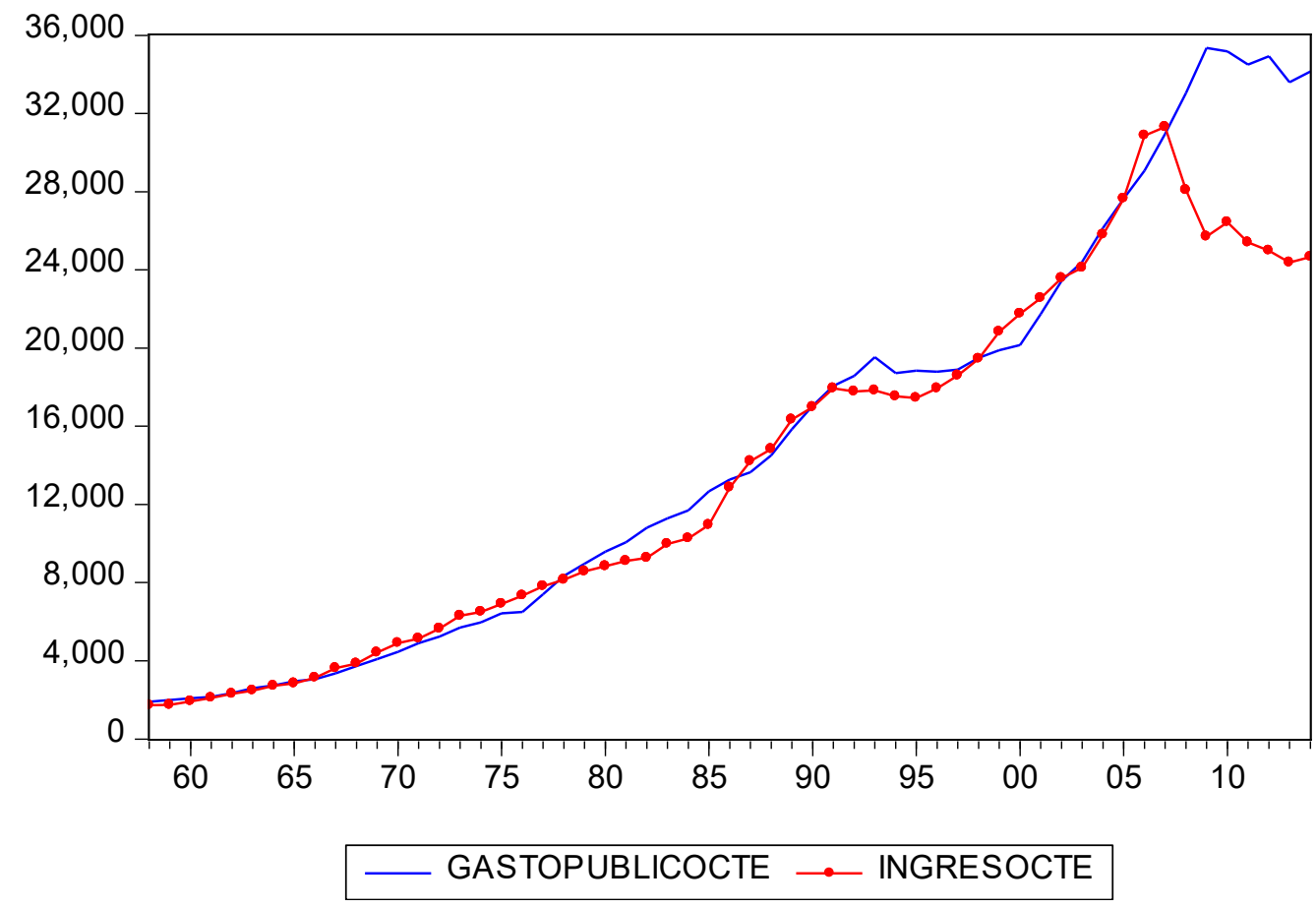

2 La base de datos utilizada está a disposición de cualquier investigador que la solicite. Correo electrónico: mjaen@ual.es. 


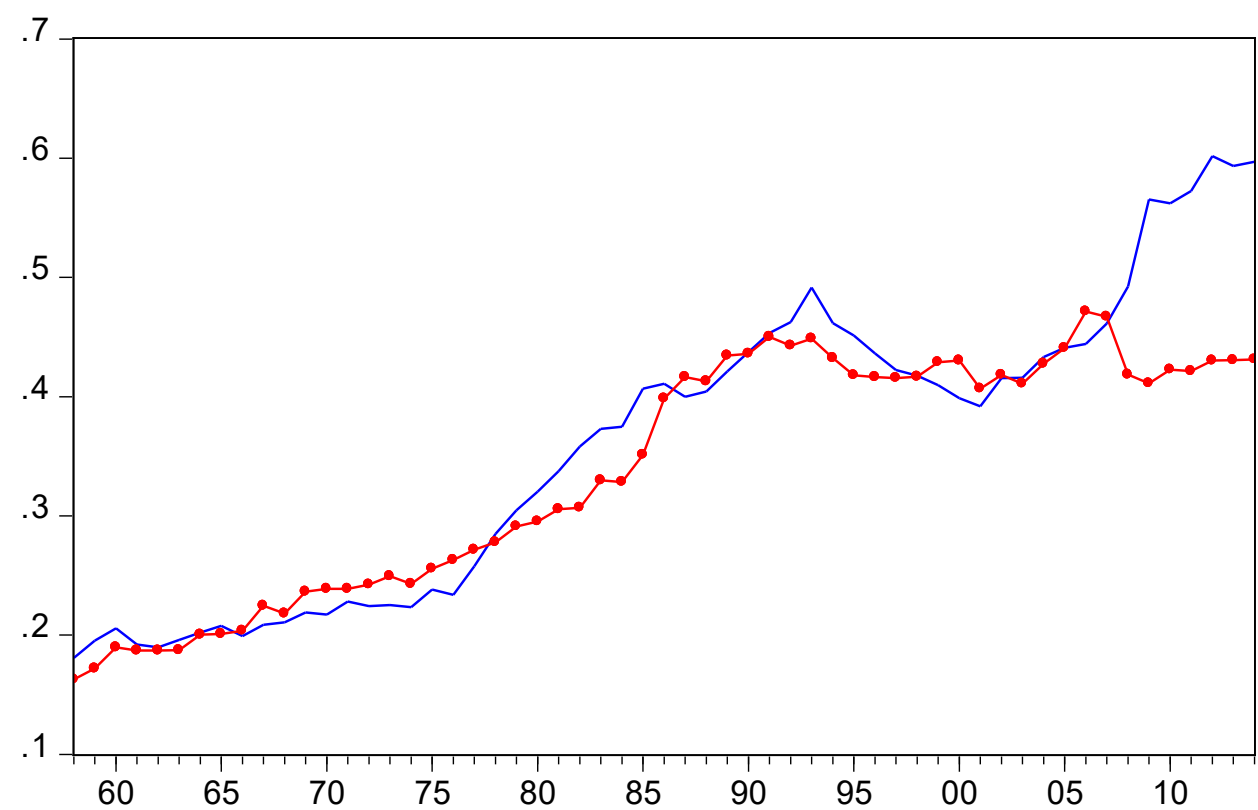

\section{$\longrightarrow$ GASTOPIB $\multimap$ INGRESOPIB}

Fuente: INE y elaboración propia

Como es habitual en la literatura, y a efectos de suavización de las series, consideramos como variables en el modelo los logaritmos del gasto público (Ln GP), el ingreso público (Ln IP) y el producto interno (o interior) bruto (Ln PIB).

Las tablas 1 y 2 muestran los estadísticos descriptivos de las tres variables, así como la covarianza y correlación entre las mismas.

La metodología utilizada consta de los siguientes pasos:

a) Contrastes de raíces unitarias de las tres series consideradas ${ }^{3}$.

b) Contrastes de cointegración.

c) Modelo de corrección de error en series cointegradas.

d) Análisis de causalidad a largo y corto plazo con el modelo de corrección de error (MCE).

3 Dada la posible existencia de cambios estructurales se han realizado los contrastes sin cambio estructural y con ellos. 
Manuel Jaén-García

Tabla 1. Estadísticos descriptivos de las tres variables

\begin{tabular}{|l|c|c|c|}
\hline & Ln GP & Ln IP & Ln PIB \\
\hline Media & 8,700588 & 8,709530 & 10,36627 \\
\hline Mediana & 9,494052 & 9,462884 & 10,38357 \\
\hline Maximo & 11,32749 & 11,19344 & 11,11418 \\
\hline Mínimo & 4,423648 & 4,688895 & 9,226209 \\
\hline Desviación Estándar & 2,292429 & 2,169174 & 0,553456 \\
\hline Asimetría & $-0,527138$ & $-0,505871$ & $-0,533201$ \\
\hline Curtosis & 1,819336 & 1,790309 & 2,283995 \\
\hline Jarque-Bera & 5,950475 & 5,906563 & 3,918455 \\
\hline Probabilidad & 0,051035 & 0,052168 & 0,140967 \\
\hline Suma & 495,9335 & 496,4432 & 590,8777 \\
\hline Suma Desviaciones cuadradas & 294,2930 & 263,4976 & 17,15357 \\
\hline Observaciones & 57 & 57 & 57 \\
\hline
\end{tabular}

Fuente: elaboración propia

Tabla 2. Correlación y covarianza entre las variables

\begin{tabular}{|l|l|c|c|c|}
\hline \multicolumn{2}{|c|}{} & Ln GP & Ln IP & Ln PIB \\
\hline \multirow{2}{*}{ Ln GP } & Covarianza & 5,16 & & \\
\cline { 2 - 5 } & Correlación & 1,00 & & \\
\hline \multirow{2}{*}{ Ln IP } & Covarianza & 4,88 & 4,62 & \\
\cline { 2 - 5 } & Correlación & 0,99 & 1,00 & \\
\hline \multirow{2}{*}{ Ln PIB } & Covarianza & 1,21 & 1,15 & 0,30 \\
\cline { 2 - 5 } & Correlación & 0,97 & 0,97 & 1,00 \\
\hline
\end{tabular}

\section{Fuente: elaboración propia}

Se puede formular la relación entre ingresos y gastos, tomando el PIB como variable de control, en la siguiente forma

$$
\begin{aligned}
& \operatorname{LnGP}_{t}=\alpha_{1}+\beta_{1} \operatorname{LnIP}_{t}+\delta_{1} \operatorname{LnPIB}_{t}+\mu_{1} \\
& \mathrm{LnIP}_{t}=\alpha_{2}+\beta_{2} \operatorname{LnGP}_{t}+\delta_{2} \operatorname{LnPIB}_{t}+\mu_{2}
\end{aligned}
$$

Donde $\mu$ es un término de error. La incorporación del PIB real al modelo se debe a que tanto el gasto público como los impuestos y otros ingresos son sensibles a 
los cambios en la actividad económica real. Por ejemplo, durante las recesiones los gastos públicos suben de forma automática (debido a las mayores prestaciones por desempleo y subsidios públicos) y los impuestos caen (debido a la disminución de las rentas personales y empresariales). Estas respuestas automáticas de las variables fiscales a las condiciones de los negocios son los elementos de estabilización automática del presupuesto. Por tanto, es importante que el PIB real sea incluido en el modelo para impedir contaminar los datos con efectos no políticos (ciclo de los negocios).

De esta forma, en la línea de Anderson, Wallace y Warner (1986), von Furstenberg, Green y Jeon (1986), Baghestani y McNown (1994), Ross and Payne (1998) y Kollias y Paleologou (2006), se incluye el PIB como variable de control en el modelo. Esta aproximación nos permite distinguir entre la relación de causalidad directa entre ingresos y gastos, y los efectos de causalidad indirecta vía PIB. Se utiliza esta aproximación, pues la mayoría de los artículos que emplean un esquema bivariante sufren del, siempre presente, problema de la tercera variable desaparecida, con la consecuencia de potenciales resultados y conclusiones inexactos.

Si gastos e ingresos están cointegrados, existe una relación a largo plazo entre ellos. Para caracterizar las relaciones de equilibrio a largo plazo y los procesos de ajuste a corto plazo entre gastos e ingresos públicos, se construye un modelo de corrección de error (MCE) en la siguiente forma ${ }^{4}$ :

$$
\begin{aligned}
& \Delta \mathrm{L} n \mathrm{GP}_{t}=\theta_{0}+\sum_{k} \theta_{1 k} \Delta{\mathrm{L} n \mathrm{IP}_{t-k}}+\sum_{k} \theta_{2 k} \Delta \mathrm{L} n \mathrm{GP}_{t-k}+\sum_{k} \theta_{3 k} \Delta{\mathrm{L} n \mathrm{PIB}_{t-k}}+\theta_{4} \mathrm{TCEI}_{t-k}+\mu_{1 t} \\
& \Delta \mathrm{L} n \mathrm{IP}_{t}=\beta_{0}+\sum_{k} \beta_{1 k} \Delta \mathrm{L} n \mathrm{IP}_{t-k}+\sum_{k} \beta_{2 k} \Delta \mathrm{L} n \mathrm{GP}_{t-k}+\sum_{k} \beta_{3 k} \Delta \mathrm{L} n \mathrm{PIB}_{t-k}+\beta_{4} \mathrm{TCE}_{t-k}+\mu_{2 t}
\end{aligned}
$$

Donde $\Delta$ es la primera diferencia del operador de retardos, $\mathrm{k}$ es la longitud del retardo. Con TCE se simboliza el término de corrección de error 5 . Es decir $\mathrm{TCEl}_{t-1}=\mathrm{L} n \mathrm{GP}_{t-1}-\alpha \mathrm{L} n \mathrm{IP}_{t-1}-\varphi \mathrm{L} n \mathrm{PIB}_{t-1}-c t e$. El parámetro $\theta_{4}$ es la velocidad de ajuste al equilibrio a largo plazo y $\mu_{1 t}$ es un ruido estadístico. E igual para la segunda ecuación.

\footnotetext{
${ }^{4}$ El modelo de corrección de error (MCE) incorpora información de las propiedades de cointegración entre las series temporales y permite, además de la unión causal del ajuste a corto plazo de las variables individuales per se, una unión causal entre dos o más variables proveniente de una relación de equilibrio (o cointegrada).

${ }^{5}$ La inclusión del TCE en el MCE da una vía extra a través de la cual se pueden producir los efectos de causalidad. Esta canal adicional de causalidad funciona a través de la corrección gradual de las variables relevantes de la desviación del equilibrio a largo plazo a través de una serie de ajustes parciales del equilibrio a corto plazo.
} 
En la tabla 3 se tiene el resultado de aplicar diferentes contrastes de raíces unitarias a las series representativas de las variables del modelo. Se observa que todas las series son integradas de orden 1.

Tabla 3. Contrastes de raíz unitaria

\begin{tabular}{|c|c|c|c|c|}
\hline Serie & $\begin{array}{c}\text { ADF test } \\
(p \text {-values })\end{array}$ & $\begin{array}{c}\text { DFGLS test } \\
\text { (VC) }\end{array}$ & $\begin{array}{c}\text { PP test } \\
(p \text {-values })\end{array}$ & $\begin{array}{c}\text { KKPSS test } \\
(\text { VC) }\end{array}$ \\
\hline LnGP & $\begin{array}{c}-0,54 \\
(0,97)^{*}\end{array}$ & $\begin{array}{c}-0,726 \\
(-3,17)^{*}\end{array}$ & $\begin{array}{c}-0,22 \\
(0,99)^{*}\end{array}$ & $\begin{array}{c}0,23 \\
(0,14)^{* *}\end{array}$ \\
\hline Ln IP & $\begin{array}{c}0,585 \\
(0,99)^{*}\end{array}$ & $\begin{array}{c}-0,6851 \\
(-3,17)^{*}\end{array}$ & $\begin{array}{c}1,34 \\
(0,99)^{*}\end{array}$ & $\begin{array}{c}0,23 \\
(0,14)^{* *}\end{array}$ \\
\hline \multirow{2}{*}{ Ln PIB } & $-2,26$ & $-1,90$ & $-0,43$ & 0,18 \\
& $(0,44)^{*}$ & $(-3,17)^{*}$ & $(0,98)^{*}$ & $(0,14)^{* *}$ \\
\hline
\end{tabular}

Notas: VC: Valor crítico

*Indica que se acepta la hipótesis nula al 5\% y ** que se rechaza

Fuente: elaboración propia

De acuerdo con las representaciones gráficas, todos los contrastes se calculan con una constante y una tendencia en la ecuación del contraste. El número de retardos para el contraste Augmented Dickey-Fuller (ADF) se calculó con el criterio de Scwartz. El ancho de banda en los contrastes Phillip-Perron (PP) y Kwiatkowski et al. (KPSS) se seleccionó con base en Newey-West con Barlett Kernell.

Tabla 4. Contrastes de raíces unitarias utilizando las primeras diferencias de las series temporales

\begin{tabular}{|c|c|c|c|c|}
\hline Serie & $\begin{array}{c}\text { ADF test } \\
(p \text {-value })\end{array}$ & $\begin{array}{c}\text { DFGLS test } \\
\text { (VC) }\end{array}$ & $\begin{array}{c}\text { PP test } \\
(p \text {-value })\end{array}$ & $\begin{array}{c}\text { KKPSS test } \\
(\text { VC) }\end{array}$ \\
\hline LnGP & $\begin{array}{c}-4,13 \\
(0,002)^{* *}\end{array}$ & $\begin{array}{c}-4,04 \\
(-1.94)^{* *}\end{array}$ & $\begin{array}{c}-4,18 \\
(0,001)^{* *}\end{array}$ & $\begin{array}{c}0,06 \\
(0,46)^{*}\end{array}$ \\
\hline Ln IP & $-3,92$ & $-3,15$ & $-3,803$ & 0,05 \\
$(-1,94)^{* *}$ & $(0,02)^{* *}$ & $(0,46)$ \\
\hline Ln PIB & $(0.01)^{* *}$ & $-2,88$ & $-4,14$ & 0,08 \\
$(-3,03$ & $(0,02)$ & $(0,02)$ & $(0,46)$ \\
\hline
\end{tabular}

Notas: VC: Valor crítico

${ }^{*}$ Indica que se acepta la hipótesis nula al 5\% y ** que se rechaza

Fuente: elaboración propia 
En línea con Perron (1989) los contrastes estándar de raíces unitarias tienden a identificar procesos estacionarios en tendencias, como estacionarios en diferencias, y pueden tener muy poco poder, incluso asintóticamente, si se ignoran los cambios de régimen. Además, durante el período estudiado se produce una fuerte convulsión social y política en España que puede haber cambiado el curso de las variables bajo estudio. Por tanto, puesto que las series pueden tener cambios estructurales se han realizado diferentes contrastes para detectarlos. En primer lugar, se usa el contraste secuencial de Bai-Perron con el que se obtienen hasta dos cambios estructurales correspondientes a los años 1997 (boom en el sector de la construcción) y 2007 (comienzo de la crisis económica y financiera). En segundo lugar, el contraste de cambio estructural de Chow rechaza la hipótesis nula de no existencia de cambios estructurales en las fechas especificadas.

Dada la existencia de estos cambios estructurales, se realizan contrastes de raíces unitarias con uno o varios cambios estructurales en momentos desconocidos en el tiempo (Zivot Andrews (ZA), Perron (P) y Lumsdaine-Papell (LP)). Las tablas siguientes muestran los resultados obtenidos.

Tabla 5. Contrastes de raíces unitarias con cambios estructurales en los datos ${ }^{6}$

\begin{tabular}{|c|c|c|c|c|}
\hline Variable & $\begin{array}{c}\text { ADF con cambios } \\
\text { estructurales } \\
(p \text {-value })\end{array}$ & $\begin{array}{c}\text { ZA test } \\
\text { (VC) }\end{array}$ & $\begin{array}{c}\text { P test } \\
\text { (VC) }\end{array}$ & $\begin{array}{c}\text { LP test } \\
\text { (VC) }\end{array}$ \\
\hline LnGP & $-3,52$ & $-3,03$ & $-3,11$ & $-3,84$ \\
$(0,37)^{*}$ & $(-4,42)^{*}$ & $(-4,83)^{*}$ & $(-6,75)^{*}$ \\
\hline Ln IP & $-4,42$ & $-3,37$ & $-2,47$ & $-2,51$ \\
& $(0,053)^{*}$ & $(-4,42)^{*}$ & $(-4,83)^{*}$ & $(-6,75)^{*}$ \\
\hline Ln PIB & $-4,17$ & $-2,84$ & $-3,03$ & $-3,66$ \\
& $(0,10)^{*}$ & $(-4,42)^{*}$ & $(-4,83)^{*}$ & $(-6,75)^{*}$ \\
\hline
\end{tabular}

Notas: VC: Valor crítico

*Indica que se acepta la hipótesis nula al 5\% y ** que se rechaza

Fuente: elaboración propia

6 En todos los casos, los puntos de cambio estructural son detectados de forma endógena por el contraste. En los dos primeros casos el contraste admite un único cambio estructural mientras el contraste LP admite dos o más cambios estructurales. Los contrastes ZA y P detectan cambios estructurales endógenos en 1983, 1987 y 2006 mientras el contraste LP lo hace en los siguientes pares de puntos 1979 y 1990, 1987 y 2006 y 1998 y 2006. En ningún caso se rechaza la hipótesis nula de raíz unitaria en las variables contra la alternativa de estacionariedad con cambio estructural en la tendencia en una fecha desconocida. La selección del número de retardos ha sido realizada utilizando el criterio de Schwartz. 
Tabla 6. Contrastes de raíces unitarias para las primeras diferencias con cambios estructurales en los datos

\begin{tabular}{|c|c|c|c|c|}
\hline Variable & $\begin{array}{c}\text { ADF con cambios estructurales } \\
(p \text {-value })\end{array}$ & $\begin{array}{c}\text { ZA test } \\
\text { (V.C. })\end{array}$ & $\begin{array}{c}\text { P test } \\
\text { (V.C.) }\end{array}$ & $\begin{array}{c}\text { LP test } \\
\text { (V.C.) }\end{array}$ \\
\hline \multirow{2}{*}{ LnGP } & $\begin{array}{c}-5,99 \\
(<0,01)^{* *}\end{array}$ & $\begin{array}{c}-5,39 \\
(-4,93)^{* *}\end{array}$ & $\begin{array}{c}-5,40 \\
(-5,23)^{* *}\end{array}$ & $\begin{array}{c}-7,57 \\
(-6,16)^{* *}\end{array}$ \\
\hline \multirow{2}{*}{ Ln IP } & $-4,73$ & $-5,18$ & $-5,04$ & $-6,97$ \\
& $(0,02)^{* *}$ & $(-4,93)^{* *}$ & $(-5,23)^{* *}$ & $(-6,16)^{* *}$ \\
\hline \multirow{2}{*}{ Ln PIB } & 5,20 & $-5,20$ & $-7,91$ & $-7,33$ \\
& $(<0,01)^{* *}$ & $(-4,93)^{* *}$ & $(-5,23)^{* *}$ & $(-6,16)^{* *}$ \\
\hline
\end{tabular}

Notas: VC: Valor crítico

${ }^{*}$ Indica que se acepta la hipótesis nula al 5\% y ** que se rechaza

Fuente: elaboración propia

De la lectura de las tablas anteriores se deduce que las tres series son I(1).

Para llevar a cabo el análisis de cointegración se usan los contrastes usuales Johansen-Juselius (JJ) sin considerar cambios estructurales. Después se consideran los cambios estructurales con dos tipos de contrastes: Gregory y Hansen (GH) en donde el cambio estructural puede considerarse tanto en la constante como en la tendencia, y Johansen-Mosconi y Nielsen (JMN), en el cual se pueden considerar uno o dos cambios estructurales en las series.

Tabla 7. Contraste de cointegración sin considerar cambios estructurales

\begin{tabular}{|c|c|c|c|c|c|}
\hline & $\begin{array}{l}\text { Número de } \\
\text { ecuaciones }\end{array}$ & $\begin{array}{l}\text { Valor propio } \\
\text { máximo }\end{array}$ & $\begin{array}{c}\text { Estadístico de la } \\
\text { traza }\end{array}$ & $\begin{array}{c}\text { Valor critico } \\
\text { al } 5 \%\end{array}$ & Probabilidad \\
\hline \multirow{6}{*}{ Contraste JJ 1} & \multicolumn{5}{|c|}{ Contraste de la traza } \\
\hline & $\mathrm{r}=0$ & 0,36 & 28,01 & 24,27 & $0,02^{* *}$ \\
\hline & $r \leq 1$ & 0,06 & 3,68 & 12,32 & $0,76^{*}$ \\
\hline & \multicolumn{5}{|c|}{ Contraste de los valores propios máximos } \\
\hline & $\mathrm{r}=0$ & 0,36 & 24,33 & 17,80 & $0,004^{* *}$ \\
\hline & $r \leq 1$ & 0,06 & 3,60 & 11,22 & $0,69^{*}$ \\
\hline \multirow{6}{*}{ Contraste JJ 2} & \multicolumn{5}{|c|}{ Contraste de la traza } \\
\hline & $r=0$ & 0,37 & 34,49 & 35,19 & $0,06^{* *}$ \\
\hline & $r \leq 1$ & 0,11 & 8,78 & 20,26 & $0,07^{*}$ \\
\hline & \multicolumn{5}{|c|}{ Contraste de los valores propios máximos } \\
\hline & $r=0$ & 0,37 & 25,71 & 22,29 & $0,02^{* *}$ \\
\hline & $r \leq 1$ & 0,11 & 6,21 & 15,89 & $0,76^{*}$ \\
\hline
\end{tabular}

Notas: La hipótesis nula $r=0$ es que no hay ningún vector de cointegración, $r \leq 1$ indica la existencia de un vector de cointegración cuando se rechaza que hay cero.

La probabilidad está incluida en la última columna.

* Indica que se acepta la hipótesis nula al 5\% y ** que se rechaza

Fuente: elaboración propia 
Johansen 1 no hay constante ni tendencia en el espacio de cointegración o vector auto-regresivo (VAR), Johansen 2 permite una constante en el espacio de cointegración y sin tendencia en el VAR. La longitud del retardo se escoge mediante el criterio de Schwartz (SIC). Se utilizan los p-valores de MacKinnnon-Haug and Michelis. Los resultados indican que la existencia de una relación de cointegración entre las variables no se rechaza ni con el contraste de la traza ni con el de valores propios máximos.

Tabla 8. Contraste de cointegración con cambios estructurales

\begin{tabular}{|c|c|c|c|c|c|}
\hline $\begin{array}{c}\text { Contraste GH } \\
\text { con CE en } \\
\text { la constante. } \\
\text { Se incluye } \\
\text { constante en } \\
\text { el conjunto de } \\
\text { datos. } \\
\text { (VC 5\%) }\end{array}$ & $\begin{array}{l}\text { Contraste GH } \\
\text { con CE en } \\
\text { la constante. } \\
\text { Se incluye } \\
\text { tendencia en los } \\
\text { datos } \\
\text { (VC 5\%) }\end{array}$ & $\begin{array}{l}\text { Contraste de la } \\
\text { traza de JMN } \\
\text { con CE en } 1997 \\
\text { y constante en } \\
\text { los datos. } \\
\text { (VC 5\%) }\end{array}$ & $\begin{array}{c}\text { Contraste de la } \\
\text { traza de JMN } \\
\text { con CE en } 1997 \\
\text { y tendencia en } \\
\text { los datos. } \\
\text { (VC 5\%) }\end{array}$ & $\begin{array}{l}\text { Contraste de } \\
\text { la traza de } \\
\text { JMN con CE } \\
\text { en } 2007 \text { y } \\
\text { constante en los } \\
\text { datos. } \\
\text { (VC 5\%) }\end{array}$ & $\begin{array}{l}\text { Contraste de } \\
\text { la traza de } \\
\text { JMN con CE } \\
\text { en } 2007 \text { y } \\
\text { tendencia en los } \\
\text { datos. } \\
\text { (VC 5\%) }\end{array}$ \\
\hline $\begin{array}{c}-3,41 \\
(-4,92)^{* *} \\
\text { CE }(2004)\end{array}$ & $\begin{array}{c}-3,73 \\
(-5,29)^{* *} \\
\text { CE }(1997)\end{array}$ & $\begin{array}{c}r=0 \quad 44,13 \\
(35,00)^{* *} \\
r \leq 1 \quad 17,63 \\
(20,30)^{*}\end{array}$ & $\begin{array}{c}r=0 \quad 32,82 \\
(34,80)^{*} \\
r \leq 16,58 \\
(20,00)\end{array}$ & $\begin{array}{c}r=062,18 \\
(35,00)^{* *} \\
r \leq 127,32 \\
(20,30)^{*}\end{array}$ & $\begin{array}{c}r=052,89 \\
(34,80)^{* *} \\
r \leq 125,39 \\
(20,00)^{*}\end{array}$ \\
\hline
\end{tabular}

Fuente: elaboración propia

En la tabla 8 se presenta los resultados obtenidos con los diferentes cambios estructurales identificados. Se utilizan los contrastes de Gregory y Hansen (GH) y Johansen, Mosconi y Nielsen (JMN). En el primer caso se realizó el contraste con constante y / o tendencia en los componentes determinísticos y que el cambio estructural se producía solo en la constante. El número de retardos se calculó con SIC y BIC. En el contraste de GH la hipótesis nula es la no cointegración mientras la alternativa es la cointegración en la presencia de un posible cambio de régimen. Se consideran los casos en los cuales la constante y el coeficiente de la pendiente tienen un único cambio estructural en un momento desconocido. El cambio estructural es detectado de forma endógena por el contraste. En el contraste JMN se consideran tendencias determinísticas lineales en los datos, y constante sin tendencia en el VAR y MCE. Se supone un cambio estructural en la constante (cambio de nivel) que se tiene en cuenta con el añadido de variables ficticias a la parte determinística del proceso. En el segundo modelo se consideran constante y tendencia en el MCE, y constante en el VAR; se supone que el cambio estructural se produce 
en la constante. En todos los casos, excepto el segundo, se rechaza la hipótesis nula de no existencia de cointegración entre las tres variables, con lo que se puede considerar que existe un vector de cointegración entre ellas.

Para analizar el cambio estructural se ha contrastado la posible igualdad a cero del coeficiente correspondiente ${ }^{7}$. En el caso de 1997 se obtiene un valor del estadístico $\chi^{2}=0,239$ con lo que no se rechaza la hipótesis nula de igualdad a cero de dicho coeficiente. En el caso de 2007 los valores del estadístico $\chi^{2}$ son 9,080 con lo que se rechaza la hipótesis nula de igualdad a cero del coeficiente.

El análisis anterior permite concluir que las variables son integradas de orden 1, I(1), y cointegradas. Por tanto, se pueden estimar las posibles relaciones de cointegración que existan entre ellas. Dada la existencia de cambios estructurales con cambios en la constante y la tendencia en algunos casos, parece adecuado analizar la causalidad a largo plazo por medio de un VAR restringido en términos de un modelo de corrección de error, pero con la adición de un término que exprese los cambios estructurales que se producen en los datos. El modelo puede escribirse como:

$$
\begin{aligned}
& \Delta \mathrm{L} n \mathrm{GP}_{t}=\theta_{0}+\sum_{k} \theta_{1 k} \Delta \mathrm{LnIP}_{t-k}+\sum_{k} \theta_{2 k} \Delta \mathrm{LnGP}_{t-k}+\sum_{k} \theta_{3 k} \Delta{\mathrm{L} n \mathrm{PIB}_{t-k}}+ \\
& \theta_{4} \mathrm{TCEl}_{t-1}+\sum_{k} \theta_{5 t} \Delta \mathrm{C}_{t-k, T 07}+\mu_{1 t}
\end{aligned}
$$

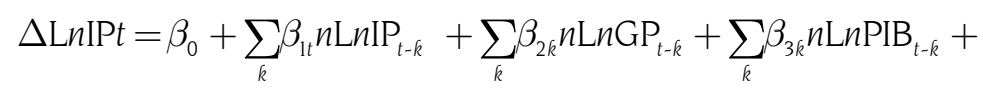

$$
\begin{aligned}
& \beta_{4} \mathrm{TCE} 2_{t-1}+\sum_{k} \beta_{5 t} n C_{t-i k \mathrm{TO} 7}+\mu_{2 t}
\end{aligned}
$$

Donde la variable dummy $\mathrm{C}_{\mathrm{t}, \mathrm{T} 7 \mathrm{7}}$ se define como igual a cero para fechas previas a los cambios estructurales, e igual a uno después. Debido a la existencia de cointegración entre ellas, al menos una de las variables debe ajustar de modo significativo a las desviaciones de la relación a largo plazo que son recogidas por $\theta_{4}$ y $\beta_{4}$ en las respectivas ecuaciones. Este parámetro describe la velocidad de ajuste hacia el equilibrio y mide la proporción de error con respecto al equilibrio que es corregido en cada etapa. Por otra parte el MCE permite establecer que el gasto causa el ingreso a largo plazo, o viceversa, siempre que el coeficiente del término de corrección de error sea estadísticamente significativo, incluso si los otros coeficientes no lo son. Un valor negativo estadísticamente significativo en los parámetros de ajuste implica causalidad a largo plazo en el sentido descrito por Engle y Granger. Para contrastar la causalidad a corto plazo se debe considerar la significación conjunta de los coeficientes de las variables retardadas. La confirmación del nexo gasto-

7 Las variables ficticias toman el valor cero para los años anteriores al cambio estructural y el valor uno a partir de dicho año. 
ingreso o al contrario es establecida cuando existe causalidad uni o bidireccional. Las tablas 9 y 10 muestran los resultados obtenidos.

Tabla 9. Términos de corrección de error ${ }^{8}$

\begin{tabular}{|c|c|c|}
\hline Variables independientes & \multicolumn{2}{|c|}{ Variable dependiente } \\
\hline & Ln GPt-1 & LnIPt-1 \\
\hline Constante & 1,80 & 0,21 \\
\hline LnGPt-1 & & 4,56 \\
& & $(6,65)^{* *}$ \\
\hline LnIPt-1 & 0,56 & \\
\hline \multirow{2}{*}{ LnPIBt-1 } & $(3,44)^{* *}$ & 2,56 \\
& 0,22 & $(2,44)^{* *}$ \\
\hline
\end{tabular}

Notas: Entre paréntesis se incluyen el estadístico t.

La hipótesis nula es la igualdad a cero de los coeficientes

*Indica que se acepta la hipótesis nula al 5\% y ** que se rechaza

Fuente: elaboración propia

Tabla 10. Coeficientes del MCE para analizar la relación de causalidad entre las variables

\begin{tabular}{|c|c|c|}
\hline Variables independientes & \multicolumn{2}{|c|}{ Variable dependiente } \\
\hline & $\Delta$ LnGPt & $\Delta$ LnGRt \\
\hline \multirow{2}{*}{$\Delta$ LnGPt-1 } & 0,22 & 0,19 \\
& $(2,02)^{* *}$ & $(0,62)^{*}$ \\
\hline \multirow{2}{*}{$\Delta$ LnIPt-1 } & 0,04 & 0,33 \\
& $(0,05)^{*}$ & $(2,30)^{* *}$ \\
\hline \multirow{2}{*}{$\Delta$ LnPIBt-1 } & 0,24 & $-0,21$ \\
& $(1,58)^{*}$ & $(-0,95)^{*}$ \\
\hline \multirow{2}{*}{$\theta 4$} & $-0,10$ & 0,02 \\
\hline \multirow{2}{*}{$\beta 4$} & $(-2,82)^{* *}$ & $(2,82)^{* *}$ \\
\hline R2 & & 0,69 \\
\hline LM & 0,53 & 96,33 \\
\hline Estadístico F & 125,07 & 38,16 \\
\hline
\end{tabular}

Notas: Entre paréntesis se incluyen el estadístico t.

La hipótesis nula es la igualdad a cero de los coeficientes

*Indica que se acepta la hipótesis nula al 5\% y ** que se rechaza

Fuente: elaboración propia

${ }^{8}$ Hay que tener en cuenta que aunque haya una sola ecuación de cointegración, al tener tres variables el coeficiente de LnIP no es el inverso del de LnGP en la otra ecuación. 
En la tabla anterior se tienen las estimaciones de los coeficientes de los términos de corrección de error de los diferentes MCE. Junto a ellos, los valores de los estadísticos t y el $\mathrm{R}^{2}$ correspondiente. El número de retardos en el MCE se calcula con AIC y SBC. La primera columna corresponde al MCE en la dirección ingreso a gasto, y la segunda, en la dirección gasto-ingreso.

Los resultados obtenidos sugieren la existencia de causalidad Granger a largo plazo de ingreso a gasto público, al nivel de significación del 5\%, con un parámetro de ajuste de $-0,1$. Se detecta, sin embargo, causalidad en el corto plazo, dado que los estadísticos de los coeficientes de los parámetros de las variables en primeras diferencias indican que estos son significativos mientras el estadístico $\mathrm{F}$ indica que son significativos de forma conjunta. En consecuencia existe causalidad bidireccional entre ingreso y gasto público a corto plazo, pero no así a largo plazo. Como se ha mencionado, los parámetros de ajuste indican la velocidad de ajuste del desequilibrio inicial hacia un equilibrio a largo plazo. En nuestras ecuaciones un 10\% de los desequilibrios son corregidos cada año.

Dado que las variables son I(1) y están cointegradas, se puede calcular la relación a largo plazo entre ellas mediante métodos de regresión mínimo-cuadráticos. Los métodos empleados son fully modified ordinary least squares (FMOLS), dynamic ordinary least Squares (DOLS) y canonical cointegration regression (CCR). Los resultados obtenidos se muestran en la tabla 9.

En ambas ecuaciones los resultados obtenidos con los tres métodos de estimación son análogos. Se observa que los parámetros tienen el signo esperado en las dos variables de referencia y todas las variables son significativas. En el primer grupo de ecuaciones la elasticidad del gasto público con respecto al ingreso es, aproximadamente, 0,3 , lo que indica que un aumento en el ingreso de un $1 \%$ posibilita un aumento en el gasto del 0,3\%.

Por su parte, la elasticidad correspondiente al PIB es aproximadamente del 0,34 lo que indicaría que un aumento del $1 \%$ en el PIB provoca un aumento del 0,34\%, es decir, el GP es inelástico a la renta. Esta última afirmación refuta, aunque sea de forma indirecta, la ley de Wagner para España, pues de acuerdo con ella el GP tiene una elasticidad renta mayor que la unidad.

Para el segundo grupo de ecuaciones la elasticidad del IP con respecto al GP está en el entorno de 0,65 con lo que un aumento del gasto del $1 \%$ posibilita un incremento del IP del 0,65\%, aunque algo mayor que en el caso contrario: el IP también es inelástico en relación al GP. En cuanto al PIB el valor de la elasticidad está en el entorno de 0,5 , con lo que un aumento del $1 \%$ en el PIB posibilita un aumento del ingreso público del $0,5 \%$. 
Tabla 11. Ecuaciones de cointegración $n^{9-10}$

\begin{tabular}{|c|c|c|c|c|c|c|}
\hline $\begin{array}{c}\text { Ecuación de } \\
\text { cointegración }\end{array}$ & LnGP & LnIP & LnPIB & D2007 & C & R2 \\
\hline FMOLS $(1)^{10}$ & & $\begin{array}{c}0,31 \\
(17,15)^{* *}\end{array}$ & $\begin{array}{c}0,34 \\
(4,60)^{* *}\end{array}$ & $\begin{array}{c}0,21 \\
(8,19)^{* *}\end{array}$ & $\begin{array}{c}2,95 \\
(4,81)^{* *}\end{array}$ & 0,99 \\
\hline DOLS (1) & $\begin{array}{c}0,30 \\
(10,74)^{* *}\end{array}$ & $\begin{array}{c}0,38 \\
(3,75)^{* *}\end{array}$ & $\begin{array}{c}0,13 \\
(2,49)^{* *}\end{array}$ & $\begin{array}{c}2,75 \\
(3,38)^{* *}\end{array}$ & 0,99 \\
\hline CCR (1) & $\begin{array}{c}0,32 \\
(17,67)^{* *}\end{array}$ & $\begin{array}{c}0,34 \\
(4,71)^{* *}\end{array}$ & $\begin{array}{c}0,21 \\
(7,67)^{* *}\end{array}$ & $\begin{array}{c}2,98 \\
(5,03)^{* *}\end{array}$ & 0,99 \\
\hline FMOLS (2) & 0,65 & & $\begin{array}{c}0,55 \\
(5,42)^{* *}\end{array}$ & $\begin{array}{c}-0,20 \\
(-6,50)^{* *}\end{array}$ & $\begin{array}{c}-2,57 \\
(-5,19)^{* *}\end{array}$ & 0,99 \\
\hline DOLS (2) & $\begin{array}{c}0,77 \\
(9,74)^{* *}\end{array}$ & & $\begin{array}{c}0,39 \\
(3,27)^{* *}\end{array}$ & $\begin{array}{c}-0,13 \\
(-3,82)^{* *}\end{array}$ & $\begin{array}{c}-2,15 \\
(-3,95)^{* *}\end{array}$ & 0,99 \\
\hline CCR (2) & $\begin{array}{c}0,65 \\
(10,63)^{* *}\end{array}$ & & $\begin{array}{c}0,56 \\
(5,71)^{* *}\end{array}$ & $\begin{array}{c}-0,20 \\
(-6,22)^{* *}\end{array}$ & $\begin{array}{c}-2,61 \\
(-5,54)^{* *}\end{array}$ & 0,99 \\
\hline
\end{tabular}

Notas: (1) considera a LnGP como variable dependiente.

${ }^{(2)}$ considera a LnIP como variable dependiente.

La hipótesis nula es la igualdad a cero de los coeficientes

Entre paréntesis se incluyen el estadístico t.

*Indica que se acepta la hipótesis nula al 5\% y ** que se rechaza

Fuente: elaboración propia

De bastante interés es el coeficiente de D2007, la variable ficticia. Para el gasto dicho coeficiente es positivo y significativo, lo que significa que en ese año, 2007, se produce un cambio estructural en la constante, y probablemente en la tendencia, en el proceso generador de datos con un aumento del gasto público. Este tiene sentido, pues en 2007 se observan los primeros efectos de la crisis inmobiliaria y financiera con un aumento del gasto público, en ese año y posteriores, que obedece a razones coyunturales (búsqueda de un aumento de la pérdida empleo producida por la crisis de la construcción) $)^{11}$. Sin embargo, dicho coeficiente es negativo, aunque significativo en el caso del ingreso público, lo que indica que en el mismo período se produce un retroceso en el ingreso público provocado por factores externos tal como la crisis inmobiliaria y la crisis general en la economía mundial con

9 Aunque no se incluye, por falta de espacio, se puede afirmar que no existe correlación entre los residuos en los tres pares de ecuaciones y que dichos residuos son estacionarios.

${ }^{10}$ A pesar de lo exiguo de la muestra, se ha realizado un análisis de robustez de los resultados considerados, con la utilización del contraste de cambio estructural de Bai-Perron, dividiendo la muestra en dos períodos: 1958-1978 y 1978-2014. Los resultados obtenidos muestran la solidez de los coeficientes de las variables tanto en signo como en magnitud.

11 Recuérdese el plan E de 2008 que tuvo un costo de 12.000 millones de euros y creó empleo, de forma transitoria, en obra pública en municipios. 
sus repercusiones en la española. Esta parálisis económica provoca, como es lógico, una disminución en los ingresos, sobre todo en los derivados de los impuestos tanto directos como indirectos.

La conclusión que se obtiene es que la influencia del ingreso en el gasto no es suficiente para provocar cambios en la política fiscal.

\section{CONCLUSIONES}

Este trabajo investiga la relación entre gastos e ingresos del Gobierno para España en el período 1958-2014. Para ello se utilizan los instrumentos de cointegración y corrección de error en un contexto de cambios estructurales, derivados de los cambios en la economía española en el período de estudio.

Los resultados obtenidos muestran que las tres variables consideradas, gasto público, ingreso público y PIB en logaritmos tienen una raíz unitaria tanto sin considerar cambios estructurales como considerando tales cambios estructurales. Los contrastes de cointegración muestran, asimismo, la existencia de cointegración con cambio estructural entre las variables. Finalmente con el uso de ecuaciones de cointegración adecuadas (fmols, dols y ccr), se obtiene una relación entre las tres variables que confirma que se puede aceptar la hipótesis ingreso gasto en el sentido de Friedman en el largo plazo, mientras en el corto se detecta una sincronización fiscal. Esta conclusión puede parecer contradictoria, aunque en el largo plazo se produce un ajuste de las políticas fiscales que puede contrarrestar la influencia en el corto plazo.

Tal como se ha relatado en el apartado 4, a partir de la crisis de 2008, se produce un retroceso en los ingresos públicos, de manera que en 2011 se sitúan en el $36,2 \%$ del PIB con un gasto del 45,6\% lo que supone un déficit fiscal del 9,4\% del PIB.

Como consecuencia, la búsqueda de una reducción del déficit público, derivada del cumplimiento del PEC y de la elevada deuda pública que se genera, que en estos momentos está en valores próximos al 100\% del PIB, implica recortes en los gastos en algunas de las partidas destinadas al Estado del bienestar, sobre todo en sanidad y educación, aunque no se ha producido un recorte real debido a la asunción por el Estado de la deuda bancaria. Esto lleva a una disminución del sector público. La repercusión de este tipo de políticas es bastante clara; de acuerdo con los datos de IGAE, en 2014 los ingresos públicos suponían el 38,6\% del PIB, mientras que los gastos eran el 44,5\%; al mismo tiempo este último ha retrocedido o se ha estancado con descensos del 0,1\% en 2011, del 2\% en 2012, del 1,6\% en 2013 con un ligero repunte del 1,7\% en 2014. 
De los resultados anteriores se puede inferir la dificultad para el Gobierno de abordar de forma adecuada los ajustes fiscales exigidos tanto por las autoridades comunitarias como por la regla establecida en el artículo 135 de la Constitución española. No se vislumbran muchas posibilidades, aunque la existencia de diversas actividades económicas que posibilitan el fraude fiscal así como la lucha contra la economía sumergida ${ }^{12}$ permitirían, si no hacer frente a las exigencias de los ajustes fiscales, al menos paliar sus consecuencias desde el punto de vista tanto del gasto corriente como de la inversión pública.

\section{BIBLIOGRAFÍA}

Anderson, W.; Wallace, M.S. y Warner, J.T. (1986). Government spending and taxation: what causes what? En: Southern Economic Journal, Vol. 52, No. 3, enero, p. 630-639.

Baghestani H. y McNown, R. (1994). Do revenue or expenditures respond to budgetary disequilibria? En: Southern Economic Journal, Vol. 61, No. 2, octubre, p. 311-322.

Barro, R. J. (1979). On the determination of the public debt. En: Journal of Political Economy, Vol. 87, No. 5, p. 940-971.

Buchanan, J. M. y Wagner, R. W. (1977). Democracy in deficit. Academic Press, New York, 223p.

Castro Fernández, F. de (2002). Una evaluación macroeconométrica de la política fiscal en España. Banco de España, Estudios Económicos No.76. 81p.

Chang, T. y Chiang, G. (2009). Revisiting the government revenue-expenditure nexus: evidence from 15 OECD countries based on the panel data approach. En: Czech Journal of Economics and Finance (Finance a uver), Vol. 59, No. 2, p. 165-172.

Chang, T.; Liu, W.R. y Caudill, S. (2002). Tax-and-spend, spend-tax or fiscal synchronization: new evidence for ten countries. En: Applied economics, Vol. 34, No. 12, p. 1553-1561.

Dahlberg, M. y Johansson, E. (1998). The revenues-expenditures nexus: Panel data evidence from Swedish municipalities. En: Applied Economics, Vol. 30, No. 10, p. 1379 86

Darrat. A. (2002). Budget balance through spending cuts or tax. En: Contemporary Economic Policy, Vol. 20, No. 3, p. 221-233.

Friedman, M. (1978). The limitations of tax limitations. En: Policy Review, No.5, p. 7-14.

Garcia Delgado J.L. (2015). Etapas y rasgos definidores de la industrialización española. En: Garcia Delgado (dir): Lecciones de Economía española, Civitas, Madrid, 448p.

Gilles, D. y Godwin, R. (2011). Testing for Multivariate Cointegration in the Presence of Structural Breaks: p-Values and Critical Values. En: Applied Economic Letters, Vol. 19, No. 16, p. 1-8.

González, M. (dir) (2013). Temas de economía española. Ed, Tirant lo Blanc libros, Valencia, España, 286p.

${ }_{12}$ La economía sumergida en España se estima alrededor del 18,5\% del PIB. Se calcula que una reducción del 5\% aumentaría la recaudación fiscal entre 17.000 y 20.000 millones de euros. 
Ho, Yh y Huang, Cj (2009). Tax-spend, spend-tax or fiscal synchronization: a panel analysis of the Chinese provincial real data. En: Journal of Economics and Management, Vol. 5, No. 2, p. 257-272.

Jaén, M. (2011). The revenues-expenditures nexus: A panel data analysis of Spain's regions. En: International Journal of Academic Research in Economics and Management Sciences, $\overline{\mathrm{Vol}}$. 1, No.1, p. 24-38.

Kollias, C. y Paleologou, S.M. (2006). Fiscal policy in the European Union. Tax and spend, spend and tax, fiscal synchronization or institutional separation? En: Journal of Economic Studies, Vol. 33, No. 2, p. 108-120.

Meltzer, A. y Richard, S. (1981). A rational theory of the size of government. En: Journal of Political Economy, Vol. 89, No. 5, p. 914-27.

Moisio, A. (2001). Spend and tax or tax and spend? Panel data evidence from Finnish municipalities during 1985-1999. VATT Discusion Papers, No. 242, 24p.

Musgrave, R. (1966). Principles of budget determination, p. 15-27. En: Cameron, H. y Henderson, W. (Eds.). Public Finance: Selected Readings. Random House, New York, 310p.

Oben, S. (2015). A causality test of the revenues-expenditures in Ghana. En: ADRRI Journal of Arts and Social Sciences, Vol. 11, No. 1, p. 1-19.

Payne, J. (2003). A survey of the international empirical evidence on the tax-spend debate. En: Public Finance Review, Vol. 31, No. 3, p. 302-324.

Peacock, A. y Wiseman, J. (1979). Approaches to the analysis of government expenditures growth. En: Public Finance Quarterly, Vol. 7, No. 1, p. 3-23.

Perron, P. (1989). The great crash, the oil price shock and the unit root hypothesis. En: Econometrics, Vol. 57, No. 6, p. 1361-1401.

Reddick, C. (2002). Canadian Provincial Budget Outcomes: a Long-Run and Short-Run Perspective. En: Financial Accountability and Management, Vol. 18, No. 4, p.355-382.

Reddick, C. y Hassan, S. (2003). Long run and short run budgeting: empirical evidence for Canada, UK and USA. En: Journal of Public Budgeting, Accounting and Financial Management, Vol. 15, No. 3, p. $\overline{354}-379$.

Roberts, P (1978). Idealism in public choice theory. En: Journal of Monetary Economics, Vol. 4, No. 3, p. 603-16.

Ross, K. y Payne, J. E. (1998). A reexamination of budgetary disequilibria. En: Public Finance Review, Vol. 26, No. 1, p.67-79.

Saunoris, J. W. (2015). The dynamics of the revenue-expenditure nexus: evidence from US state government finances. En: Public Finance Review, Vol. 43, No. 1, p. 108 134.

Saysombath, P y Kyophilavong, P. (2013). The causal link between spending and revenue: The Lao PDR. En: International Journal of Economics and Finance, Vol. 5, No. 10, p. 111-116.

Serrano, J. M. y Bandrés. E. (2015). Sector público. En: García, J. L. y Myro, R. (eds): Lecciones de economía española, Civitas-Thomson Reuters, Madrid, 448p. 
Tamames, R. (2005): Introducción a la economía española. Alianza Editorial, Madrid, 576p.

Von Furstensberg, G. M.; Green, R. J. y Jeong, J. H. (1986). Tax and spend or spend and tax? En: The Review of Economics and Statistics, Vol. 68, No. 2, p.179-188.

Wagner, R.E. (1976). Revenue structure, fiscal illusion and budgetary choice. En: Public Choice, Vol. 25, No. 1, p.30-61.

Wildavsky, A. (1988). The new politics of budgetary process. Glenview, Scott Foresman, 468p. 